\title{
Session 7
}

\section{ICMEs in the heliosphere}

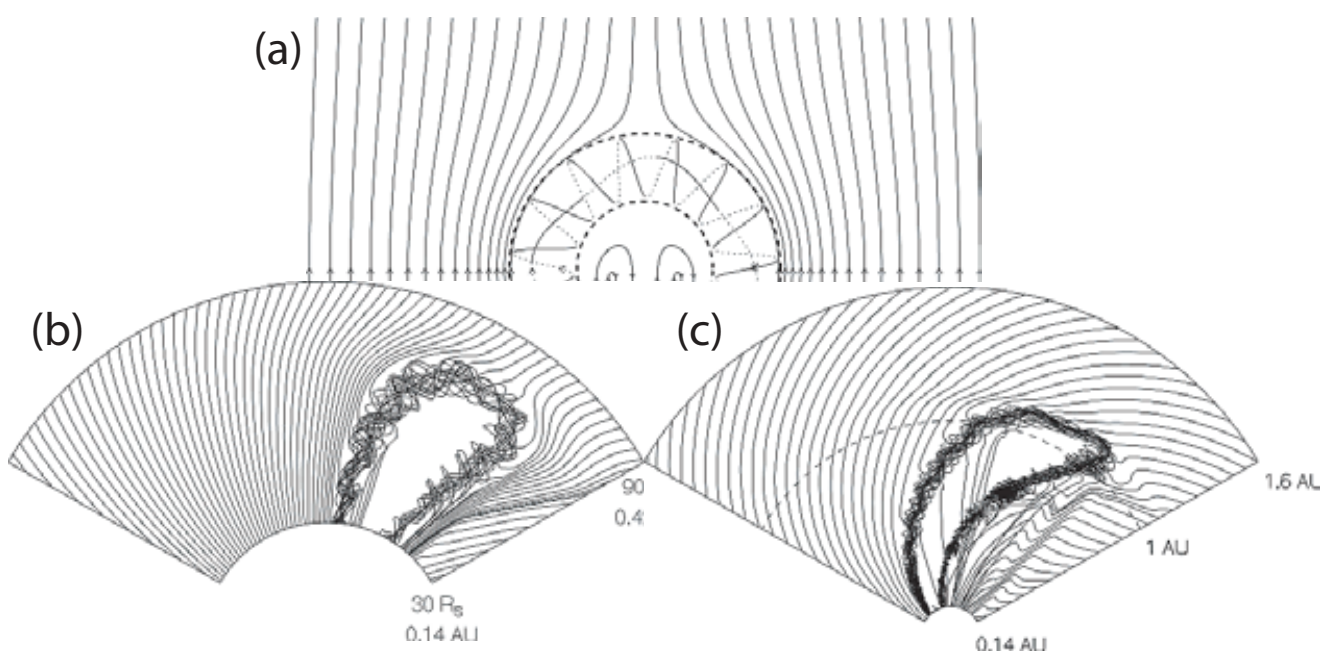




\title{
Modeling Interplanetary Coronal Mass Ejections
}

\author{
Pete Riley \\ Science Applications International Corporation, San Diego, California 92121. USA. \\ email: pete.riley@saic.com
}

\begin{abstract}
Heliospheric models of Coronal Mass Ejection (CME) propagation and evolution provide an important insight into the dynamics of CMEs and are a valuable tool for interpreting interplanetary in situ observations. Moreover, they represent a virtual laboratory for exploring conditions and regions of space that are not conveniently or currently accessible by spacecraft. In this review I summarize recent advances in modeling the properties and evolution of CMEs in the solar wind. In particular, I will focus on: (1) the types of ICME models; (2) the boundary conditions that are imposed; (3) the role of the ambient solar wind; (4) predicting new phenomena; and (5) distinguishing between competing CME initiation mechanisms. I will conclude by discussing what topics will likely be important for models to address in the future.
\end{abstract}

Keywords. Sun: coronal mass ejections, magnetohydrodynamics, solar wind

\section{Introduction}

In this report I summarize recent advances in modeling the properties and evolution of Coronal Mass Ejections (CMEs) in the solar wind. I will describe the current state of research and suggest what topics will likely be important for models to address in the future. I will focus on the physics described by the models and not specifically on the models themselves. Given the need for brevity, references will be selective and illustrative, rather than comprehensive. Other reviews that complement the present one have been given by Linker et al. (2002); Cargill \& Schmidt (2002); Riley (1999). While I emphasize fluid and MHD modeling in this report, it should be noted that other modeling approaches have been used with success. The extension of force-free flux rope fitting (Lepping et al. 1990) to include the effects of expansion (Osherovich et al. 1993; Marubashi 1997) and multiple spacecraft (Mulligan et al. 2001), for example, have allowed further classification of this important subset of CMEs. Hybrid codes have also been used to model the interaction of fast CMEs with the ambient solar wind allowing ion-kinetic effects to be explored (Riley et al. 1998).

Since the basic mechanism(s) by which CMEs erupt at the Sun is (are) not well known (Forbes 2000; Klimchuk 2001), it is therefore not surprising that models developed to investigate the initiation and evolution of CMEs both near the Sun and in the solar wind tend to be idealized. In fact, to make problems tractable, significant approximations must be made. For example, consider the placement of the inner radial boundary. For many years, this was chosen to be beyond the outermost critical point (e.g., Hundhausen \& Gentry (1968); Dryer et al. (1989); Riley et al. (1997b); Odstrcil \& Pizzo (1999b,c,a); Cargill \& Schmidt (2002); Vandas et al. (2002)). Modeling CME propagation and evolution beyond this point is a much simpler task than including the initiation process and evolution through the lower corona. Given accurate boundary conditions at say $20-30 R_{S}$, the physics of the medium is simpler and better understood, and the MHD equations used to describe the system are easier to solve. Further, the minimum time step required 
to advance the solutions are also typically much larger than would be required if the lower corona were included. Unfortunately, it is difficult to measure the plasma and magnetic field properties in this region, leading to the specification of ad hoc boundary conditions. Moreover, such an approach completely avoids the question of CME initiation.

A second, often used approximation is to neglect the magnetic field (e.g., Hundhausen \& Gentry (1968)). Thus strictly speaking the simulations are valid only for high- $\beta$ CMEs. The characteristic speeds at which pressure disturbances propagate in the simulation is less than in the real solar wind, and magnetic forces. Obviously such studies cannot address questions related to the magnetic structure of the CME. Nevertheless, they have proven to be extremely useful in illuminating the fundamental aspects of the processes by which both transient and corotating disturbances evolve in the solar wind (see, for example, reviews by Hundhausen (1985); Gosling (1996)).

Currently there is a trend toward "modular" modeling of space-plasma systems, where several specialized codes are integrated together, with the output from one model providing the input to the next model (e.g., http://ccmc.gsfc.nasa.gov). In some cases, such as the ionosphere-magnetosphere system, this can lead to a complex feedback loop. On the other hand, the coupling of solar coronal models with interplanetary models is considerably simpler owing to the supersonic nature of the flow at the boundary (Odstrcil et al. 2002).

Algorithms are constantly being updated to include more and more realistic physics. The methods of solution are also being improved on to take advantage of new developments in numerical techniques as well as new computing paradigms. Adaptive Mesh Refinement (AMR), for example, is a technique that allows both large- and small-scale structure to be resolved within a single simulation (e.g., Odstrcil et al. (2002); Manchester et al. (2004a)). The Message Passing Interface (MPI) is an approach that allows one to utilize a large number of processors simultaneously, leading to simulations at significantly higher resolution than previously possible.

\section{Basic features of ICME models}

The basic features of ICME models can be broken down into several areas, including: (1) the equations, physics, and hence approximations that are intrinsic to the model;

(2) the imposed boundary conditions; (3) the ambient solar wind into which the CME propagates; and (4) the mechanism for initiating the CME.

\subsection{The Physics in the Models}

Generally speaking, all of the models solve some variation of: Ampere's law, Faraday's law, Ohm's law, mass continuity (conservation of mass), the equation of motion (conservation of momentum), and an energy equation (energy conservation).

In the hydrodynamic approximation the magnetic field terms are omitted and the equations are significantly simpler to solve. In some of the models the resistivity is set to zero, simplifying Ohm's law, and reducing the system to the ideal MHD limit. Some of the heliospheric models also neglect the gravity term in the equation of motion; a reasonable approximation for regions above $20-30 R_{S}$.

An important distinction between the models is how the energy equation is treated. Setting the energy source term to zero reduces it to a simple polytropic relationship. This may be a reasonable first approximation for the solar wind, where the measured adiabatic index, $\gamma$, for the protons is about 1.5 (Totten et al. 1996); however, it is questionable for the solar corona, which is nearly isothermal. Moreover, models that couple the corona and heliosphere must account for the discontinuous change in $\gamma$ between the two models 
(Odstrcil et al. 2002). Several solutions to these problems have been proposed. Roussev et al. (2003), for example, recently implemented a $\gamma$ that was a function of distance from the Sun, being $\sim 1$ at the Sun and approaching 5/3 further out. While this is an apparently workable practical solution, there is little physically to justify it, and, more importantly, no way to assess how the fall-off with distance should occur. Alfvén waves have also been incorporated to heat the corona and/or drive the acceleration, at least for ambient solar wind (e.g., Usmanov et al. (2000)). This might appear to be more physically justifiable, however, it is unlikely that Alfvén waves acting alone are sufficient, and thus, one is in effect, replacing one set of free parameters with another. The most promising long-term solution would appear to be the treatment of more complicated energy equation, including the effects radiation, thermal conduction, coronal heating, and resistive and viscous diffusion. While this is a challenging problem, progress is being made (Lionello et al. 2001).

\subsection{Boundary Conditions}

The heliosphere is naturally a spherical (or cylindrical in 2-D) region so most models solve the $(\mathrm{M}) \mathrm{HD}$ equations in this system (note, however, that for computational reasons, some codes, e.g. BATS-R-US (Manchester et al. 2004a) are implemented in Cartesian coordinates). Thus at a minimum, boundary conditions at the inner spherical surface (the inner radial boundary) and outer spherical surface (the outer radial boundary) must be considered. In 1-D models, these are simply points. Some models focus on a more limited region of space, typically by reducing the coverage in latitude (e.g., Odstrcil et al. (2002)). The outer boundary is simple since it is always super-sonic and super-Alfvénic. Thus whatever reaches this point (plasma, fields, waves, etc) must leave the system. The same holds true for the inner boundary if it is chosen to be beyond the outermost critical point. We refer to these models as "super-critical" and those that include the lower corona as "sub-critical" models (the outer boundary of the sub-critical models may stretch out far into the solar wind). This distinction is important insofar as the physics and methods of solution are considerably simpler for the super-critical models. Moreover, the lack of any substantial observations at this heliocentric distance provides little in the way to constrain the models.

\subsection{The Ambient Solar Wind}

The medium into which CMEs propagate plays a substantial role in the subsequent evolution of ejecta and their associated disturbances. The simplest possible background wind is a single-speed wind. For 1-D models, this is really the only type of wind you can prescribe. The next level of sophistication is to set up a two-state wind consisting of slow, dense wind at low latitudes, and fast, hot, tenuous wind at high latitudes (Riley et al. 1997b). This mimics the declining phase and solar minimum picture that Ulysses observed during its first orbit (e.g. Riley et al. (1997a)), and for the purposes of running idealized, generic simulations is perfectly adequate. This wind pattern can be prescribed in two dimensions (Riley et al. 1997b) or as a "tilted-dipole" configuration in three dimensions (Odstrcil \& Pizzo 1999b,c,a). An example of this wind pattern (at $30 R_{S}$ ) is shown in Figure 1(a). However, to address the evolution of ICMEs for specific events, we need to specify the actual ambient solar wind that was present at the time of the eruption of the CME. An example of a more realistic wind is shown in Figure 1 (b). The speed was computed using the SAIC coronal MHD model (Riley et al. 2001b) and has been shown to reproduce the essential large-scale features of the solar wind, particularly during relatively quiet solar conditions. 

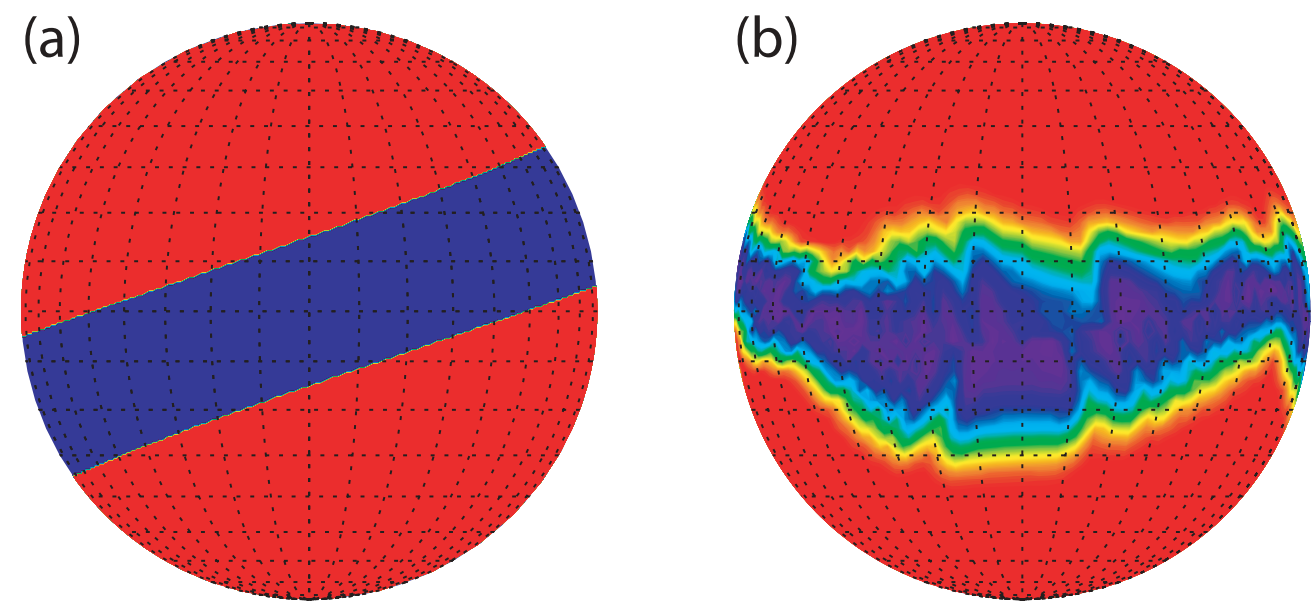

Figure 1. Ambient solar wind speed at $30 R_{S}$ for: (a) an idealized "tilted-dipole" configuration; and (b) a specific time period during solar minimum conditions (Carrington rotation 1913). Blue corresponds to the slowest speeds $(400 \mathrm{~km} / \mathrm{s})$ while red corresponds to the fastest speeds $(750$ $\mathrm{km} / \mathrm{s})$.

\subsection{CME Initiation}

Once the ambient medium has been prescribed, the CME can be "initiated". There are a number of approaches for accomplishing this, but they can be broadly separated into those that actually model the initiation process (i.e., self-consistent models) and those that simply pass a representation of a CME through the inner boundary (i.e., ad hoc models). The oldest - but still used - example of the latter is to set up a "pulse" in one or more of the plasma and/or magnetic field parameters (e.g., Riley (1999)). This could be a strong speed change, a density and/or temperature change, or some combination (e.g., Hundhausen \& Gentry (1968)) or could include a coherent magnetic structure, such as a flux rope (e.g., Vandas et al. (2002)). One of the appealing aspects of these ad hoc pulses is that it allows the modeler to explore a wide range of the parameter space. Input conditions can be modified to better reproduce a specific set of observations. Of course the danger is that while "tweaking" the inputs in this way can produce better model results, you do not necessarily learn much from it.

The self-consistent models attempt to model the actual eruption process of the CME. As such they have inherently more scientific value. On the other hand, it can be very difficult to explore CME evolution in the solar wind for different eruption processes, as these models currently only produce a limited range of properties. From a computational standpoint, these types of models require considerably more resources than the pulse-type simulations. Whereas a 1-D simulation incorporating a simple pulse might takes minutes on a workstation computer, a 3-D global MHD solution might require weeks of time on a multi-processor supercomputer. Thus there is a place for both types of approaches, depending on the specific problem being addressed.

\section{Examples of ICME models}

It would be impossible to comprehensively review all of the CME modeling work that has been undertaken in the last 40 years. Instead, I have chosen several specific examples that: (1) illustrate the main general aspects of the models that were described above; and (2) have been used to address a specific scientific problem. 

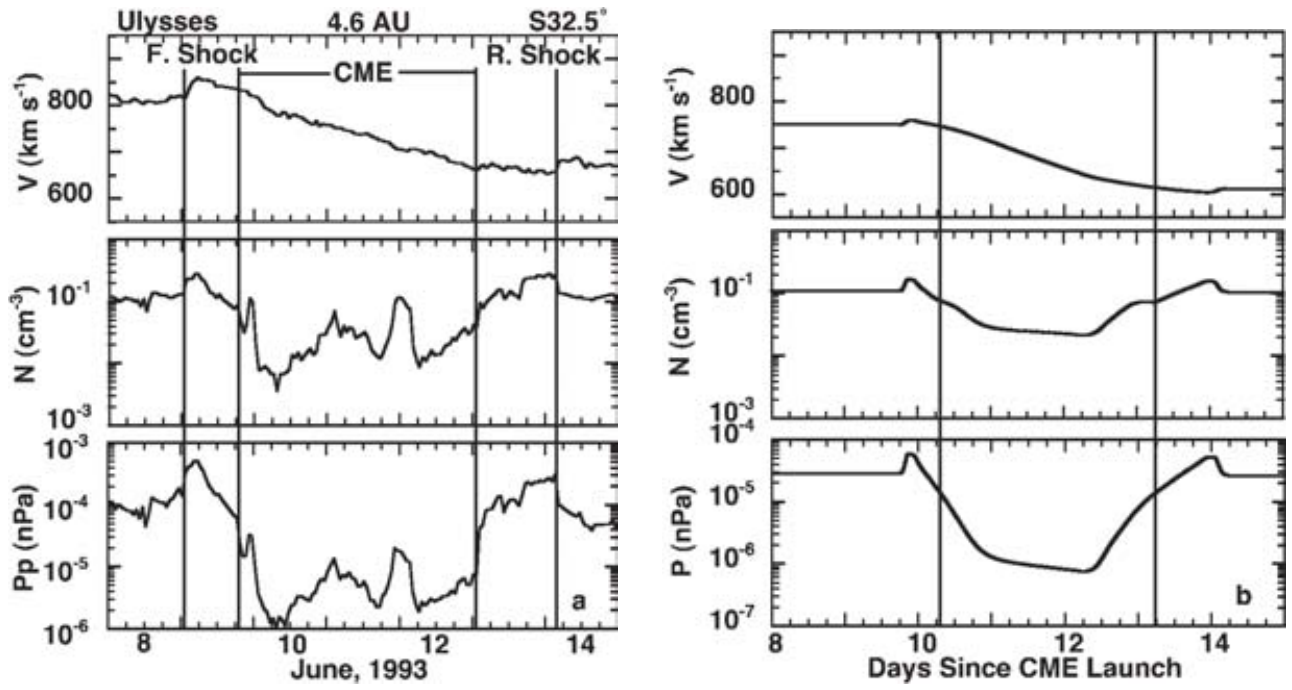

Figure 2. Comparison of Ulysses observations of a high-latitude ICME with results from a 1-D, super-critical, hydrodynamic model. Adapted from Gosling et al. (1998).

\subsection{One-dimensional, super-critical hydrodynamic models}

Let us start by discussing the simplest of all ICME models. These are: (1) super-critical, meaning the inner radial boundary is at 20-30 $R_{s} ;(2)$ idealized, single-flow solar wind; and (3) initiated by an ad hoc pulse. They are simple to implement and the results are easy to interpret. However, there is no way to include a magnetic field in a meaningful way and there will always be lingering questions about the uniqueness of the solution. On the other hand, they have been, and continue to be useful in interpreting in situ observations, providing clues about the evolution of the ejecta. As a example, in Figure 2 we compare Ulysses observations of an ICME at high heliographic latitudes, with the results of such a simulation. The ICME was observed while Ulysses was located at $4.6 \mathrm{AU}$ and 32.5 degrees south latitude. Vertical lines indicate the location of the ICME. A forward and reverse shock bound the ejecta on either side. These are both fast-mode shocks. In the rest frame of the ambient plasma, the forward shock is propagating away from the Sun and the reverse shock is propagating towards the Sun. However, in the spacecraft's frame, everything is being convected away from the Sun. The general features of this event are: (1) relatively symmetric density and temperature profiles; (2) a declining speed profile (indicating that the ejecta is expanding); and (3) a decrease in the density and pressure. The inferences we draw from these profiles was that an initially high-pressure ejecta drove forward and reverse waves away from it that subsequently steepened into shocks.

The results of the 1-D, gas-dynamic simulation are shown on the right-hand side of Figure 2. After an initial equilibrium was established (with values that match what was observed at Ulysses), a pulse was initiated whereby the speed was dropped monotonically over a 10 hour period, while at the same time, the density and thus pressure were enhanced in a bell-shaped profile. How were these values chosen? Well, it is not unreasonable to expect a CME to be launched with an internal pressure higher than the ambient, although it is likely that in reality, this pressure is supplied by the magnetic field. The speed decrease merely reflects what was observed; the speed before the CME was high and lower after it.

Not surprisingly, the simulated profiles compare well with the observations. The declining speed, decrease in density and pressure are all reproduced. Even the sheath region 
and shocks match well. The standoff distance is not as great in the simulation because the characteristic speed is the sound speed and not the fast mode speed as it is in reality. Even the asymmetry in the locations of the shocks is reproduced. This is an evolutionary effect; the leading edge of the CME is younger than the trailing edge, when viewed at a single point in space.

So on one hand, we have successfully modeled a high-latitude ejecta from 30 Rs to 4.6 AU, and provided a plausible picture of the ejecta's evolution during this interval. And, they have been successfully applied to a number of problems, including: the evolution of CMEs are large heliocentric distances (Riley \& Gosling 1998); the acceleration of CMEs near the Sun (Gosling \& Riley 1996); and the relationship between density and temperature within CMEs and its implications for the polytropic index of the plasma (Riley et al. 2001a). However, many fundamental questions remain unanswered, such as: How was the CME initiated? What was it's early evolutionary history? What role did the magnetic field play? How does this localized picture related to the global structure of the event? To answer these questions, we must increase the dimensionality of the simulations, include the magnetic field, and consider the triggering mechanism.

\subsection{3-D, super-critical MHD models}

It has long been known that the particular trajectory taken by a spacecraft through a CME and its associated disturbance can radically alter the observed profiles. In fact, the recent classifications of CMEs in to "simple" and "complex" (Burlaga et al. 2001) may be, at least in part, a consequence of such observational selection effects. Marubashi (1997) illustrated how a single event could be seen by one spacecraft as a non-fluxrope CME while at another it would appear as a magnetic cloud, suggesting that the delineation between magnetic clouds (or flux ropes) and CMEs may be an artificial one. CMEs simultaneously observed by Ulysses and ACE had such different profiles at the two spacecraft, that only by using global MHD modeling could we confidently infer that the events were one and the same (e.g., Riley et al. (2003)).

To illustrate the use of 3-D, super-critical MHD models let us consider the model developed by Vandas et al. (2002), who simulated the evolution of a flux-rope CME from $30 R_{S}$ out to $1 \mathrm{AU}$ within an idealized single-speed wind. The initial state of the magnetic cloud was that of a section of a torus, who's foot points were tied to the Sun. The model output is summarized at 3 times in Figure 3.

The top panel shows the flux rope embedded within the ambient solar wind field at the beginning of the simulation. The speed, density, and temperature of the torus were exactly the same as the ambient solar wind; only the magnetic field structure was different. Thus no shocks or significant disturbances would be expected to form. After emerging half of the torus, the boundary conditions were held constant, except that they were rotated in azimuth to simulate the effect of solar rotation. The two lower panels show the magnetic field in the equatorial plane at two times following the emergence of the torus. On the left, at 32 hours, the flux rope is beginning to develop some structure associated with rotation. By 120 hours, the apex of the flux rope has reached $1 \mathrm{AU}$ and flattened considerably.

A particularly interesting aspect of this study was the construction of simulated time series at various longitudinal positions through the ejecta. For example, a spacecraft intercepting the apex of the flux rope would subsequently encounter the flux rope legs. The encounter with the apex displayed the usual signatures of a flux rope CME: field enhancement, rotation in the field, a modest speed decrease, indicating expansion, and a decrease in temperature and density. The encounter with the CME legs also showed characteristic signatures in the magnetic field, with no corresponding plasma signatures. 


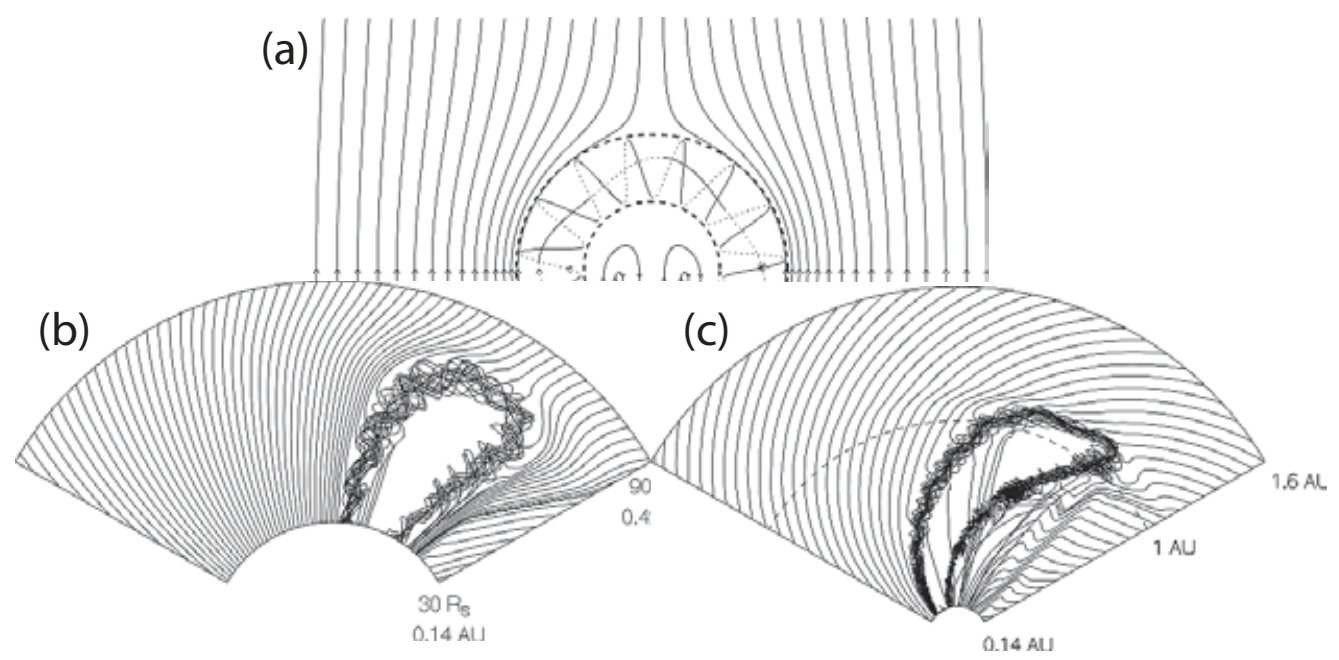

Figure 3. Results from 3-D, super-critical, MHD model. (a) Initial conditions for the flux rope and ambient solar wind; (b) Magnetic field lines at 32 hours; and (c) magnetic field lines at 120 hours.

Other more complex profiles were presented, which depended on the spacecraft's location. Some observations of "double flux ropes" (e.g., Vandas et al. (1993)), could be cases where a spacecraft flew through the same ICME twice.

\subsection{3-D, Sub-Critical ICME models}

As we have noted, modeling the solar environment below the critical points is more complicated because information can now travel in both directions. Nevertheless several groups are modeling the Sun's extended Corona from $1 R_{S}$ to $1 \mathrm{AU}$, and beyond. Wu et al. (1999), for example, generated a CME from the eruption of a helmet streamer using an ad hoc increase in the azimuthal component of the magnetic field. The University of Michigan group (e.g., Groth et al. (2000b); Manchester et al. (2004a); Roussev et al. (2004)) have developed a finite-volume, AMR scheme to study CME evolution from the Sun to Earth. The CME is "initiated" in one of several ways. Groth et al. $(2000 \mathrm{~b}, \mathrm{a})$ applied a localized density enhancement at the solar surface, essentially mimicking a pressure pulse. In contrast, Manchester et al. (2004a,b) superimposed the magnetic and density solutions of the 3-D Gibson \& Low (1998) flux rope within the coronal streamer belt; the CME being driven by the resulting force imbalance.

The University of Michigan model is summarized in Figure 4. In this simulation, a two-state wind (aligned to the rotation axis) is constructed. The Gibson-Low flux rope is superimposed onto the background field. Thus the eruption process takes place because the system begins out of equilibrium. As with the simulations initiated beyond the super-critical points, "inserting" a CME near the solar surface and allowing it to evolve from that point allows enormous freedom to adjust the initial parameters to fit the observations. It may turn out that for the purposes of space weather prediction, such an approach is the most practical. A particular set of observations, for example, may suggest an appropriate initial configuration and perturbation to produce a CME that reproduces observations near Earth.

It is unlikely, however, that such approaches will uncover the underlying eruption mechanism(s). Instead, the free parameters must be constrained by developing more self-consistent models. Towards this goal, Linker \& Mikić (1997) initiated an eruption through differential rotation and followed its evolution out to 1 AU. Later, Odstrcil et al. 
(a)

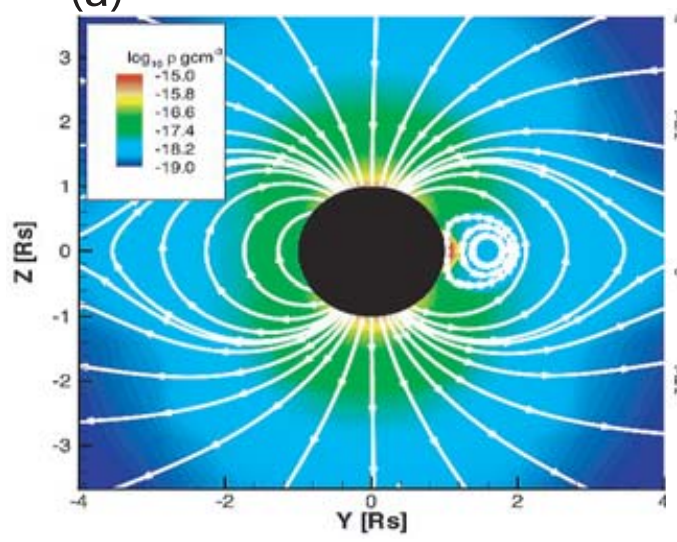

(b)
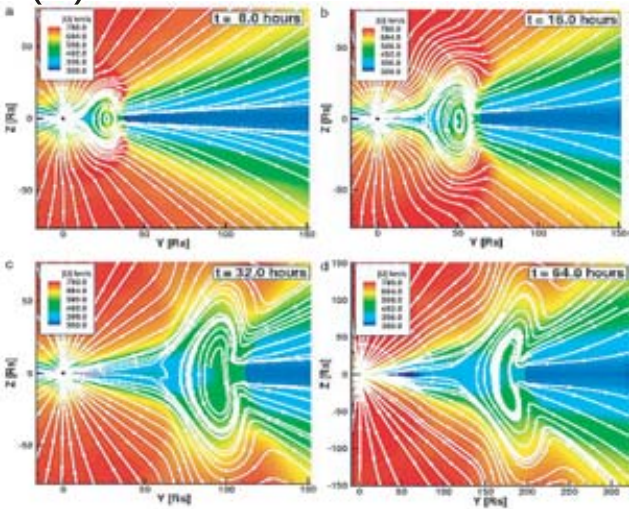

Figure 4. Simulation results from a 3-D, sub-critical, MHD model. (a) Initial conditions; and (b) Four snapshots at 8, 16, 24, and 32 hours following the launch of the CME.

(2002) coupled this coronal model with a heliospheric model to simulate the eruption of a CME at the Sun and its propagation out to 5 AU. In spite of the idealized nature of the eruption process and ambient solar wind, the solution was remarkably rich and complex. Riley et al. (2003), for example, used these results to interpret the plasma and magnetic field signatures of a CME observed by both ACE and Ulysses, which were aligned in longitude, but separated significantly in radial distance and latitude. Favorable comparisons with in situ observations, however, do not necessarily validate one eruption mechanism. Differentiating signatures are needed to do this (see below).

\subsection{Model Predictions}

The simulations by Odstrcil et al. (2002) also suggested that a jetted outflow, driven by post-eruptive reconnection underneath the flux rope occurs and may remain intact out to $1 \mathrm{AU}$ and beyond (Riley et al. 2002). Comparison between simulations and observations of a magnetic cloud with similar signatures suggested that velocity and/or density enhancements observed trailing magnetic clouds may be the signatures of such reconnection, and not associated with prominence material, as has previously been suggested.

Figure 5 shows the relationship between this velocity enhancement and the ejecta as the flux rope approaches $1 \mathrm{AU}$. This is a view in the meridional plane from a point just below the equatorial plane. The color contours show the radial velocity, while the arrows summarize the meridional magnetic field. Superimposed are several field lines. These are the helical field lines making up the flux rope. Note how the velocity enhancement has remained intact because of the umbrella-like protection provided by the ejecta plowing into the solar wind ahead as well as the quiet, slower wind that is following the disturbance: the velocity pulse is immersed in an expansion wave (or rarefaction region). Note also that the reconnection feature has a very limited angular extent, suggesting that such a signature might be difficult to observe. A preliminary analysis of WIND observations of magnetic clouds suggests that perhaps $5 \%$ of events may contain such a feature. Gosling et al. (2004) have recently provided a detailed analysis of a number of cases suggestive of reconnection in the solar wind. In principle, they may be associated with this phenomenon.

If these results are further substantiated, they may provide us with a unique window into the reconnection process. Ultimately, these types of comparisons with observations may provide constraints on mechanisms for the initiation of CMEs. At present there is 


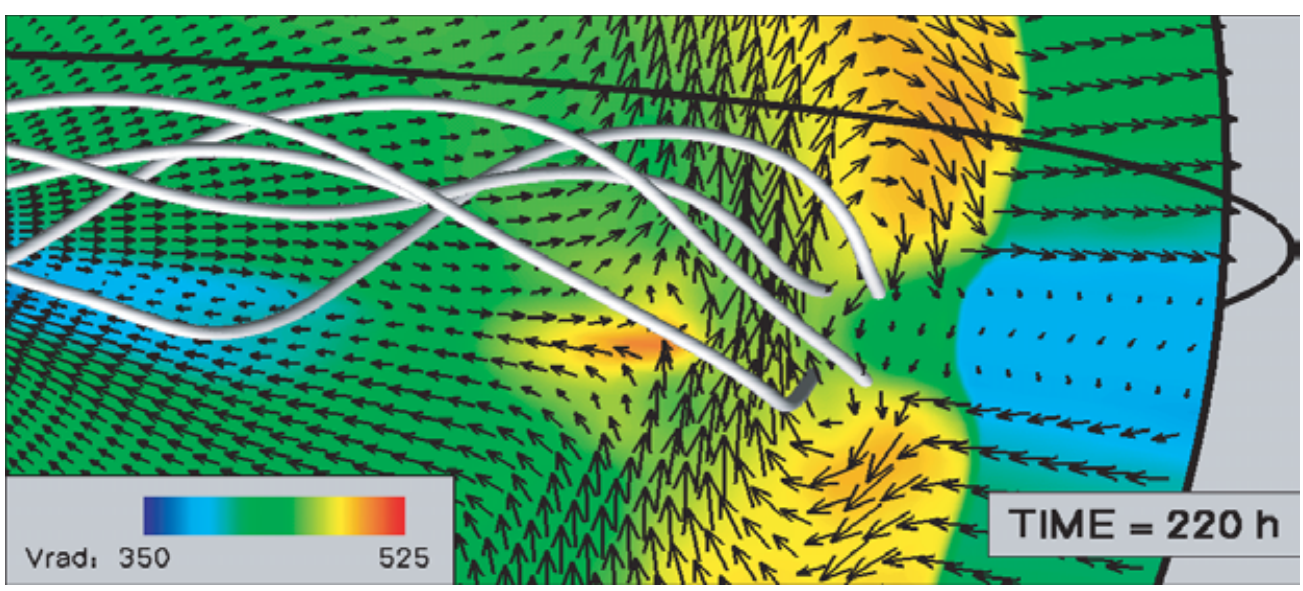

Figure 5. Results from a 3-D, sub-critical MHD simulation of a flux rope erupted at the Sun via flux cancellation as it approaches $1 \mathrm{AU}$. The color contours show radial velocity and the arrows indicate the direction and magnitude of the magnetic field line the meridional plane. A selection of magnetic field lines threading the flux rope have been drawn. The solid black curve indicates the trajectory of Earth.

very little to constrain the models: Quite conveniently, the models do not yet predict any distinguishing features that we can test against.

\subsection{Differentiating between CME initiation models}

A fundamental problem in solar physics is how CMEs erupt. Many of the pieces of the puzzle have been assembled, but how they relate to one another, and what role each one plays is not known. We know, for example, that the energy for the eruption is supplied by the magnetic field. Observationally, we know that many CMEs contain flux ropes. Yet we do not know during which phase of the eruption the flux rope forms, nor do we know why some ICMEs contain flux ropes while others do not.

A number of theoretical models have been proposed (see reviews by Forbes (2000); Klimchuk (2001). Each model addresses - and is therefore consistent with - some subset of the observed properties of CMEs. Not surprisingly, few of them are in direct conflict with any of the observations. Thus it has proven difficult to distinguish between them. As an example, in Figure 6 I compare the "flux cancellation" model (Linker et al. 2001) with the "breakout" model (Antiochos et al. 1999). The models distinguish themselves primarily by the underlying structure of the region producing the eruption. In principle, the breakout model can erupt either via photospheric shear or flux cancellation. The distinguishing feature of the breakout model is the requirement of a more complex quadrupolar configuration. In addition to the differences in the large-scale magnetic field configuration, the main difference between the two models is that photospheric shear alone was used to trigger the eruption in the breakout model. The outstanding difference between the two is that a single flux rope structure is produced in the flux cancellation eruption, whereas a "double flux rope" appears to have been generated in the breakout model. This leading "flux rope" is not the same helical structure that is produced as the main ejecta since it contains essentially no azimuthal field component. It results from reconnection at the leading edge of the ejecta, together with reconnection of the streamer belt further ahead of the event. The picture is, however, quite suggestive, and if further substantiated, may provide an explanation for some of the double flux ropes that have been observed in the solar wind (e.g., Vandas et al. (1993)). On the other hand, these 

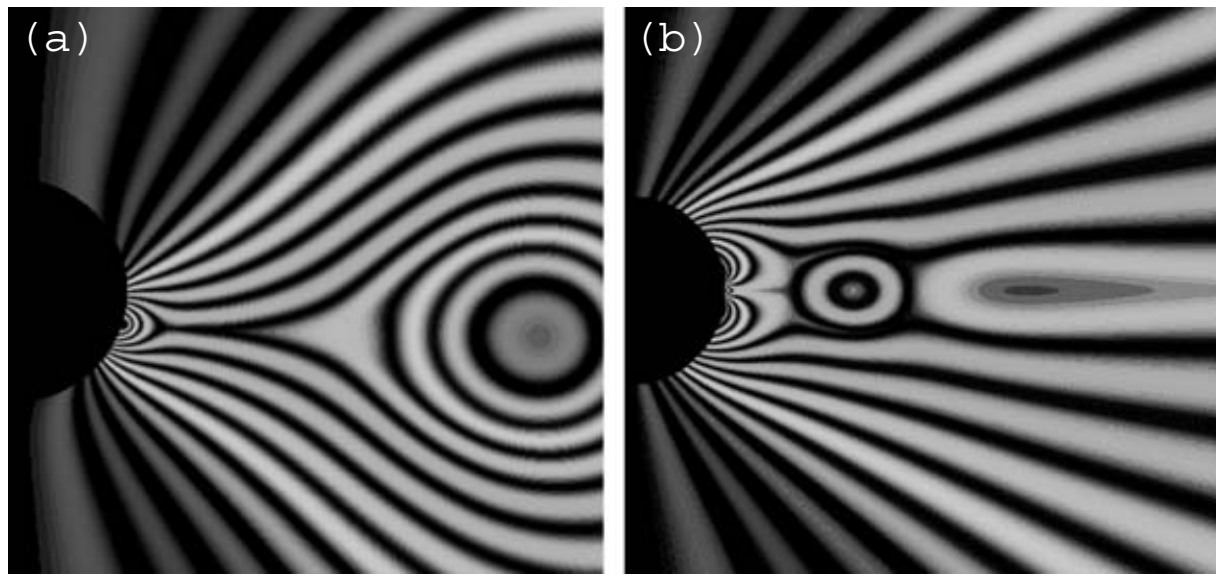

Figure 6. Comparison of (a) flux cancellation and (b) breakout eruption profiles.

are preliminary results and should not be viewed as definitive. In particular, that most ICMEs do not appear to have this double rotation should not be used to invalidate the breakout model. One feature that does appear to be robust, however, is the requirement for reconnection to proceed at the leading edge of the erupting flux rope in the breakout model. Indeed, it is this reconnection that generates the necessary force imbalance to allow the CME to be able to escape the confines of the overlying field in the first place. Thus while we expect reconnection to occur in both models underneath the erupting flux rope, we would also expect to see reconnection at the leading edge of CMEs erupting via the breakout scenario. Observationally, this might appear as a discontinuity in the sheath region between the shock and the flux rope. Discontinuities, however, are very common occurrences in the solar wind.

\section{Closing Remarks and Future Research}

In this review I have illustrated how models are helping us to understand the formation and evolution of CMEs in the solar wind. I would like to reiterate that these remain idealized simulations. Yet even as such, they are full of interesting phenomena. They allow us to interpret localized in situ measurements within a global perspective, and they also provide clues for interpreting remote solar observations, such as LASCO, EIT, and soft x-ray observations. Most importantly they allow us to connect these two disparate datasets. The simulations can be used to explore the role played by the ambient solar wind on the distortion of the ejecta and its associated disturbance, and they can be used to predict new phenomena that may or may not be present in the data and so eventually be used to constrain models of CME eruption.

Even a cursory glance at these results shows that the simulations are not adequately represented by cartoons typically drawn for CMEs in the solar wind. The cross section of ICMEs has been shown, for example, to be strongly distorted away from circular, a result simply of CME expansion in a spherical geometry (Riley \& Crooker 2004).Given the strong interaction of CMEs with the ambient solar wind, we must view pictures of CMEs deduced from single point spacecraft measurements with caution. For example, it is unlikely that the so-called "over-expanding" CMEs are really isolated bubbles in the high speed solar wind as has been drawn (Gosling et al. 1994).

The models are far from perfect and it is important to read the "fine print". The availability of sophisticated visualization tools today has led to the production of amazing 
pictures and movies that, by their very complexity, convey the idea that everything has been solved. In reality, the important approximations and assumptions have been hidden from the audience. In many cases, these limitations may be playing a crucial role in the model. The old adage of "buyer beware" should be adhered to.

Predicting the path of future research is clearly speculation, undoubtedly driven, at least in part, by one's current interests. Nevertheless, it may be of some use to list several likely topics that may be pursued in the upcoming years. One challenge will undoubtedly involve the ability to self-consistently model CMEs with a range of properties. How do we initiate slow and fast CMEs, for example? Are they generated by the same mechanism, or are there two (or more) mechanisms that are responsible? Self-consistent models currently can only produce flux-rope CMEs. What are the underlying differences between these and CMEs that don't contain a flux rope? Is it a observational selection effect or are there intrinsically different mechanisms for producing each type?

A important new area in CME research concerns the acceleration and transport of energetic particles. Roussev et al. (2004), for example, have used the inferred properties of a CME-driven shock to argue that diffusive shock acceleration theory can account for solar energetic protons with energies below $10 \mathrm{GeV}$. Several groups (e.g., Sokolov et al. (2004)) are developing and coupling particle acceleration and transport codes with global MHD models to address which mechanisms are important for the production of SEPs.

We may be entering a new era of CME modeling. In the future, we will see models becoming increasingly capable of modeling specific events. This will require capabilities to accurately reproduce a disparate set of remote and solar observations. We may see some of these models transition from research tools to operation tools, capable of predicting the onset of geo-effective phenomena (although see Cargill \& Schmidt (2002) for a more conservative opinion on this).

It is important to remember that these models are only tools that allow us to better understand CME phenomena. To close, then, we provide an illustrative selection of questions that we may be able to answer using the models described here. What are the fundamental evolutionary distinctions between CMEs and magnetic clouds? What topology is predicted for CMEs in the heliosphere by various initiation mechanisms? What is the relationship between the 3-part structure of CMEs as seen in coronagraph observations and their interplanetary counterparts? What processes control the solar connectivity of field lines embedded within CMEs? How do the properties of the ambient solar wind modify the evolution of the ejecta? How does the internal magnetic structure of a flux rope affect its distortion in the solar wind? How do in situ signatures (as would be seen by a spacecraft) change depending on where the simulated CME is sampled? What differentiating observational signatures do the models predict?

\section{Acknowledgements}

The author gratefully acknowledges the support of the National Aeronautics and Space Administration (Living with a Star Program) and the National Science Foundation (SHINE Program) in undertaking this study.

\section{References}

Antiochos, S. K., Devore, C. R., \& Klimchuk, J. A. 1999, Astrophys. J., 510, 485

Burlaga, L. F., Skoug, R. M., Smith, C. W., Webb, D. F., Zurbuchen, T. H., \& Reinard, A. 2001, J. Geophys. Res., 106, 20957

Cargill, P. J., \& Schmidt, J. M. 2002, Ann. Geophys., 20, 879 
Dryer, M., Detman, T. R., Wu, S. T., \& Han, S. M. 1989, THE INTERNATIONAL HELIOSPHERIC STUDY: COSPAR27, 75

Forbes, T. G. 2000, J. Geophys. Res., 105, 23153

Gibson, S., \& Low, B. 1998, Astrophys. J., 493, 460

Gosling, J. T. 1996, Annual Review of Astronomy and Astrophysics, 34, 35

Gosling, J. T., McComas, D. J., Phillips, J. L., Weiss, L. A., Pizzo, V. J., Goldstein, B. E., \& Forsyth, R. J. 1994, Geophys. Res. Lett., 21, 2271

Gosling, J. T., \& Riley, P. 1996, Geophys. Res. Lett., 23, 2867

Gosling, J. T., Riley, P., McComas, D. J., \& Pizzo, V. J. 1998, J. Geophys. Res., 103, 1941

Gosling, J. T., Skoug, R. M., McComas, D. J., \& Smith, C. W. 2004, Accepted for publication in J. Geophys. Res.

Groth, C. P. T., de Zeeuw, D. L., Gombosi, T. I., \& Powell, K. G. 2000a, J. Geophys. Res., 105, 25053

—. 2000b, Adv. Space Res., 26, 793

Hundhausen, A. J. 1985, IN: Collisionless shocks in the heliosphere: A tutorial review, 34, 37

Hundhausen, A. J., \& Gentry, R. A. 1968, Astron. J., 73, 63

Klimchuk, J. A. 2001, in in Space Weather Geophys. Monogr., Vol. 125 (Washington, DC: AGU), 143

Lepping, R. P., Jones, J. A., \& Burlaga, L. F. 1990, J. Geophys. Res., 95, 11957

Linker, J. A., Lionello, R., Mikić, Z., \& Amari, T. 2001, J. Geophys. Res., 106, 25165

Linker, J. A., \& Mikić, Z. 1997, Coronal Mass Ejections, 99, 269, edited by N. Crooker, J. Joselyn, and J. Feynmann, p. 269, AGU, Washington, D. C.

Linker, J. A., Mikić, Z., Riley, P., Lionello, R., \& Odstrcil, D. 2002

Lionello, R., Linker, J. A., \& Mikić, Z. 2001, Astrophys. J., 546, 542

Manchester, W., Gombosi, T., Roussev, I., De Zeeuw, D., Sokolov, I., Powell, K., Tóth, G., \& Opher, M. 2004a, Journal of Geophysical Research (Space Physics), 109, 1102

Manchester, W., Gombosi, T., Roussev, I., Ridley, A., De Zeeuw, D., Sokolov, I., Powell, K., \& Tóth, G. 2004b, Journal of Geophysical Research (Space Physics), 2107

Marubashi, K. 1997, in Coronal Mass Ejections (Geophys. Monogr.), ed. N. U. Crooker, J. A. Joselyn, \& J. Feynman, Vol. 99 (Washington, DC: AGU), 147

Mulligan, T., Russell, C. T., Anderson, B. J., \& Acuna, M. H. 2001, Geophys. Res. Lett., 29, 4417

Odstrcil, D., Linker, J. A., Lionello, R., Mikić, Z., Riley, P., Pizzo, V. J., \& Luhmann, J. G. 2002, J. Geophys. Res., 107, DOI 10.1029/2002JA009334

Odstrcil, D., \& Pizzo, V. J. 1999a, J. Geophys. Res., 104, 28255

—. 1999b, J. Geophys. Res., 104, 483

—. 1999c, J. Geophys. Res., 104, 493

Osherovich, V. A., Farrugia, C. J., \& Burlaga, L. F. 1993, Adv. Space Res., 13, 57

Riley, P. 1999, in Solar Wind Nine Proceedings of the Ninth International Solar Wind Conference, ed. S. R. Habbal, R. Esser, V. Hollweg, \& P. A. Isenberg, Vol. 471 (Nantucket, MA: The American Institute of Physics (AIP Conference Proceedings)), 131

Riley, P., Bame, S. J., Barraclough, B. L., Feldman, W. C., Gosling, J. T., Hoogeveen, G. W., McComas, D. J., Phillips, J. L., Goldstein, B. E., \& Neugebauer, M. 1997a, Adv. Space Res., 20, 15

Riley, P., \& Crooker, N. 2004, Astrophys. J., 600, 1035

Riley, P., \& Gosling, J. T. 1998, Geophys. Res. Lett., 25, 1529

Riley, P., Gosling, J. T., \& Pizzo, V. J. 1997b, J. Geophys. Res., 102, 14677

—. 2001a, J. Geophys. Res., 106, 8291

Riley, P., Linker, J., Mikić, Z., Odstrcil, D., Zurbuchen, T., Lario, D., \& Lepping, R. 2003, Journal of Geophysical Research (Space Physics), 108, 2

Riley, P., Linker, J. A., \& Mikić, Z. 2001b, J. Geophys. Res., 106, 15889

Riley, P., Linker, J. A., Mikić, Z., Odstrcil, D., Pizzo, V. J., \& Webb, D. F. 2002, Astrophys. J., 578,972

Riley, P., Omidi, N., \& Gosling, J. T. 1998, in American Geophysical Union, Fall Meeting 1998 (San Francisco, CA: AGU), abstract 
Roussev, I., Gombosi, T., Sokolov, I., Velli, M., Manchester, W., DeZeeuw, D., Liewer, P., Tóth, G., \& Luhmann, J. 2003, Ap. J. Letters, 595, L57

Roussev, I., Sokolov, I., Forbes, T., Gombosi, T., Lee, M., \& Sakai, J. 2004, Astrophys. J. Lett., 605, L73

Sokolov, I. V., Roussev, I. I., Gombosi, T. I., Lee, M. A., Kota, J., Forbes, T. G., Manchester, W. B., \& Sakai, J. I. 2004, submitted to Ap. J. Lett.

Totten, T. L., Freeman, J. W., \& Arya, S. 1996, J. Geophys. Res., 101, 15629

Usmanov, A. V., Goldstein, M. L., Besser, B. P., \& Fritzer, J. M. 2000, J. Geophys. Res., 105, 12675

Vandas, M., Fischer, S., Pelant, P., \& Geranios, A. 1993, J. Geophys. Res., 98, 21061

Vandas, M., Odstrčil, D., \& Watari, S. 2002, J. Geophys. Res., 107, 2

Wu, S., Guo, W., Michels, D., \& Burlaga, L. 1999, J. Geophys. Res., 104, 14789

\section{Discussion}

ShiBATA: What is the largest distance from the sun of the post-eruption reconnection? Did you find the signature of slow shocks in the post-eruption reconnection?

RILEY: Most of the reconnection takes place during and shortly after the eruption of the CME. But, undoubtedly, this reconnection continues for some time. I would have to re-analyze the simulation results to see how long the reconnection persisted at detectable levels. We haven't looked for the evidence of slow shocks in the post-eruption reconnection, but I would be surprised if we find such a signature.

SCHWEnN: Do the models give any hint on how the 3-part (or multi-part) structure near the sun is transformed into the 2-part structure in IP space?

RILEY: The models do provide a good connection between the cavity and the flux rope in interplanetary space. Because we do not realistically model the prominence/filament, we can only infer its mapping to the trailing edge of the ICME. However, it is the loop (or bright front) that has the most ambiguity. The coronal structure suggests that much of this structure existed prior to its propagation but it is difficult to assess what contribution comes from swept-up material. This is something we are currently investigating.

RufFOLO: How can you observationally identify when you go through the near-radial field of the legs of a CME?

RILEY: The near-radial fields are quite easy to identify in the in-situ data. To make the association with CMEs requires ancillary information. For example, you might pass through a shock and compressed plasma before encountering the radial fields. You might also see some counterstreaming suprathermal electrons. Alternatively, remote solar observations might indicate the passage of a CME past your point of observation within the right time frame.

SCHMIEDER: Is the work done by the Belgium group relevant to your work concerning the modelization of ICMEs?

RILEY: Yes it is, but I had no time to review all the simulations of ICMEs.

Kouтснмy: Doing numerical simulations, is it not possible to include what seems important from the observational point of view: filament/prominence eruption, heating of the prominence gas, mass loading? 
RILEY: These are obviously important effects, and to a limited extent, and with varying degrees of success, they have been addressed within the framework of more limited modeling. Including these effects in the global MHD models I have described here will be quite a challenge, but is something that we are in the process of addressing.

ZHUKOV: Is there any progress in modeling the north-south interplanetary magnetic field component $\mathrm{Bz}$ in ICMEs?

RILEY: Yes! 